\title{
Propulsion characteristics of wing-in-ground effect dual-foil propulsors
}

\author{
Pengfei Liu ${ }^{\mathrm{a}, *}$, Tao Wang ${ }^{\mathrm{b}}$, Guojun Huang ${ }^{\mathrm{c}}$, Brian Veitch ${ }^{\mathrm{d}}$, Jim Millan ${ }^{\mathrm{a}}$ \\ ${ }^{a}$ Institute for Ocean Technology, National Research Council Canada, 1 Arctic Avenue, P.O. Box 12093, St. John's, NL, Canada, A1B 3T5 \\ ${ }^{\mathrm{b}}$ College of Naval Architecture and Ocean Engineering, Dalian University of Technology, China \\ ${ }^{\mathrm{c}}$ Institute of Mechanics, Chinese Academy of Sciences, 15 Bei-Si-Huan-Xi-Lu, Beijing, 100080, China \\ ${ }^{\mathrm{d}}$ Faculty of Engineering and Applied Science, Memorial University of Newfoundland, St. John's, NL, Canada, A1B 3X5
}

\section{A R T I C L E I N F O}

\section{Article history:}

Received 28 August 2009

Received in revised form

28 January 2010

Accepted 11 March 2010

Available online 3 April 2010

\section{Keywords:}

Oscillating foil

Propeller

Propulsor

Wing-in-ground effect

Panel method

\begin{abstract}
A B S T R A C T
Propulsion characteristics of wing-in-ground effect propulsors were investigated using a comparative analysis of thrust and powering characteristics between wing-in-ground (WIG) effect thrusters and traditional screw propellers. WIG thrusters were found to have constant thrust production and efficiency, nearly independent of speed of advance, as contrary to screw propellers, whose optimum efficiency occurs at only one speed point. To produce the same amount of thrust as equivalent screw propellers, WIG thrusters have to work under heavily loaded operating conditions. WIG thrusters were also found to produce a relatively lower but nearly constant efficiency and thrust, independent of speed. Another distinguishing propulsion characteristic revealed for WIG thrusters is that they are capable of operating at much higher speeds, in a range of three to six times that of screw propellers of the same size. While the speed range of screw propellers is mainly limited by their geometric pitch, the speed range of WIG thrusters has no speed limit in ideal fluid. In reality, the speed range is only limited by viscous drag and cavitation, or compressibility, in water or air, respectively. This suggests a potential for WIG thrusters of higher speed application than screw propellers. An experimental investigation and validation of the propulsion system is warranted.
\end{abstract}

Crown Copyright @ 2010 Published by Elsevier Ltd. All rights reserved.

\section{Introduction}

Unlike single-foil oscillating propulsors in which thrust is created by motions with a combination of both pitch and heave, WIG thrusters create thrust mainly by heave-only motion. A WIG thruster does not only have a simpler motion control mechanism due to the absence of the pitch control [1] but is also able to produce about four times the thrust and about $20 \%$ higher efficiency than a single-foil heave-only propulsor at design speed [2]. The heave-only motion without pitch control also eliminates side force and moment implications. While thrust force of conventional screw propellers can only be transmitted along the shaft of propellers, WIG thrusters produce thrust force perpendicular to their shafts, which can be directed at an arbitrary angle through a relative rotation between the thruster core unit and its casing [3]. By adding a damped pitching flexibility (finite torsional stiffness) to the shaft, the self-controlled pitch can be automatically adjusted by flow around the foil section to provide a substantially reduced separation and stall angle at the start-up speed [3]. These advantages suggest a

\footnotetext{
* Corresponding author. Tel.: +1 709772 4575; fax: +1 7097722462 .

E-mail address: Pengfei.Liu@nrc.ca (P. Liu).
}

feasible propulsor alternative to screw propellers, especially for manoeuvring or high-speed applications of underwater objects. They also suggest a further investigation on loading and powering characteristics for engineering design. Having developed and used a time-domain unsteady multiple-body boundary element method, (a code named dual-foil oscillating foil boundary element method, or DF_OSFBEM), we obtained the thrust production and powering characteristics of the wing-in-ground effect thruster and performed a propulsion similitude analysis along with a comparison of the findings that will lead to a unique WIG thruster application and design. A detailed description of the multi-body, unsteady panel method is presented in [3].

The wing-in-ground effect thruster is a relatively new unconventional propulsor. A schematic diagram of a two-foil WIG thruster is shown in Fig. 1. The wing-in-ground effect is created when the pivot shafts of the two foils perform a counterphase heave-only motion, i.e., moving towards and apart from each other [1].

In the two- or three-dimensional case, the horizontal axis or plane of symmetry, respectively, is the pseudo "ground", created by the motion of the two foils being a mirror image of each other. This is analogue to an insect's flight with the wings flapping toward and apart from each other in an anti-phase motion. Single oscillating foils (SOFs) as a propulsor have been studied extensively 


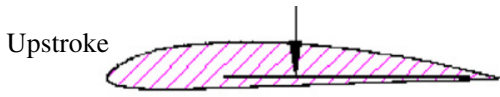

Heave amplitude $\mathrm{h}$

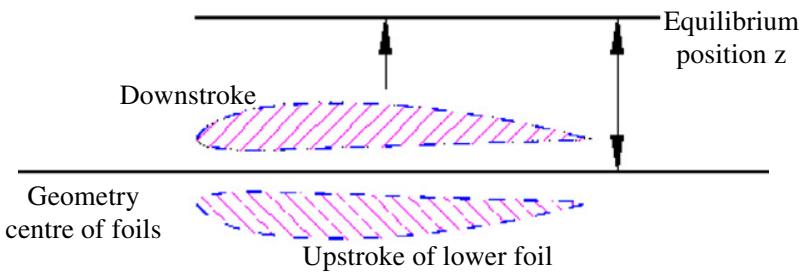

Downstroke of lower foil

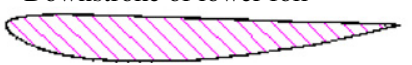

Fig. 1. A schematic of the two foil sections of the wing-in-ground (WIG) effect thruster. Two oscillating foils move towards and apart sinusoidally in counter phase.

(e.g., [4-6]). However, SOFs are not usually feasibly applicable in marine and aeronautical applications because of their complicated motion control requirement for both pitch and heave and their much lower thrust production, though at a very lightly loaded condition a set of flexible fin whale flukes could achieve an efficiency of up to $90 \%$ [5]. In Fig. 1 for a WIG thruster, to avoid the two foils colliding with each other at the close up stroke, the equilibrium position $z$ is set larger than the heave amplitude $h$ at about $10 \%$ chord. The shaft is set with infinite torsional stiffness so the pitch angles of the foils at the close up position are at about zero. As mentioned above, the mechanical advantage of WIG thrusters over the SOFs is the absence of pitch control so the side force and momentum of the WIG thruster cancel each other. A parametric study for geometry and motion parameters was conducted by Liu [2] and the investigation showed that the WIG effect can create as much as 3.7 times more thrust than a single oscillating foil heave-only propuslor and achieve an extra $20 \%$ of efficiency at the design speed at a reduced frequency $k$ of about 0.75 . Viscous flow propulsive performance evaluation was also carried out in an analysis of a 2D WIG thruster by Wang et al. $[7,8]$, in terms of propulsive performance at low Reynolds numbers and trailing edge vortices and flow separation.

A conceptual WIG thruster unit for marine application is shown in Fig. 2. The produced thrust force points at the foil's trailing edge in alignment with the chord line, normal to the line that links the shafts of the two foils. This is different from conventional screw propellers in which the thrust force acts along only the propeller shaft (forward and reverse).

The thrust vector of the WIG thruster, by rotating the core thruster unit, in grey in Fig. 2, relative to the casing, in blue, can be manipulated at an arbitrary angle, in $0-360^{\circ}$ [3]. Using this feature, an array of WIG effect thrusters, when properly controlled, can achieve a manoeuvrability of 6-degrees of freedom motion simultaneously. Fig. 3 shows a marine WIG thruster prototype drawing. While the main motor drives the heave motion of the dual foils, a smaller motor serves for the angular positioning of the thruster casing and hence the thrust vector.

\section{Validation and comparison of propulsive characteristics of several oscillating foil configurations}

Validations of the code and convergence studies on this timedomain panel method were performed for both single oscillating foil and dual-foil WIG flapping propulsors; verification and

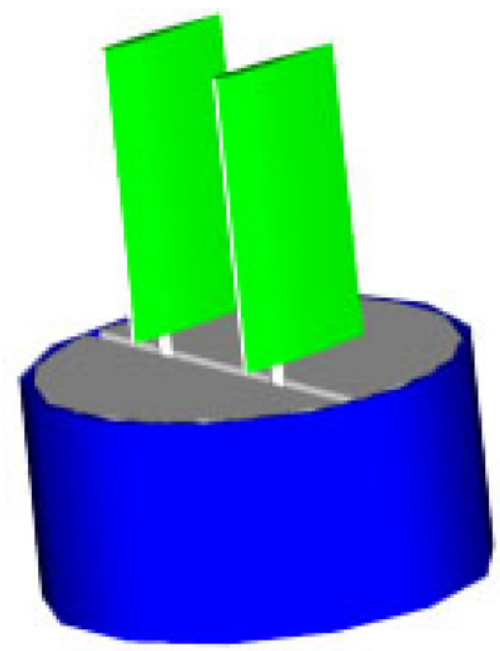

Fig. 2. A conceptual 3D drawing of a marine WIG thruster unit. The thruster unit core (in grey) can rotate relative to the outside casing (in blue) to produce $0-360^{\circ}$ of controllable thrust vector. (For interpretation of the references to colour in this figure legend, the reader is referred to the web version of this article.)
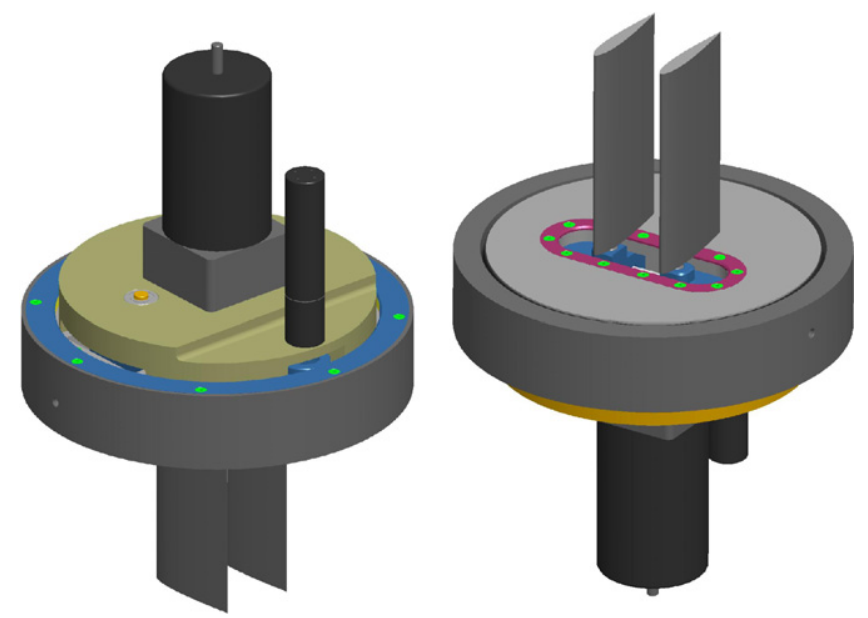

Fig. 3. A marine WIG thruster prototype unit designed by Fast Prototyping Division, Design and Fabrication Services in Ottawa, National Research Council Canada.

comparison between the method and experimental measurements were performed previously as well [5,2]. Figs. 4-6 show the thrust coefficient measured [1] and predicted using a two-dimensional panel method [1] and predicted by DF_OSFBEM for dual aerial foils oscillating in a counter phase to create a wing-in-ground effect (WIG). The detailed geometry and motion parameters of the aerial foil of an aspect ratio of 9.375 were given in [3].

The predicted thrust by DF_OSFBEM agreed well with the measurements for all three oscillating frequencies. Figs. 4-6 show a common trend that DF_OSFBEM slightly under-predicted thrust under very lightly loaded conditions (i.e., very high speed region) and over-predicted thrust at very low speeds (i.e., under very heavily loaded condition). Only for the highest oscillating frequency of $8 \mathrm{~Hz}$ at a very low forward speed of $3.00 \mathrm{~m} / \mathrm{s}$, is the discrepancy between measured and predicted thrust performance substantial. At this heavily loaded condition, the maximum effective angle of attack (AoA) is about $23^{\circ}$. This is an extremely large effective AoA for a 2D foil, under which severe stall and flow separation are unavoidable. For a two-dimensional foil in steady flow at a Reynolds number of $10^{6}$ the threshold of the effective AoA to cause a stall is about $12^{\circ}$ (see [9]). However, for an unsteady oscillating foil, stall and separation will be delayed, which 
Thrust coefficients $\mathrm{Ct}$ of one of the dual foils at a frequency of $4 \mathrm{~Hz}$, estimated maximum AoA: $12^{\circ}$

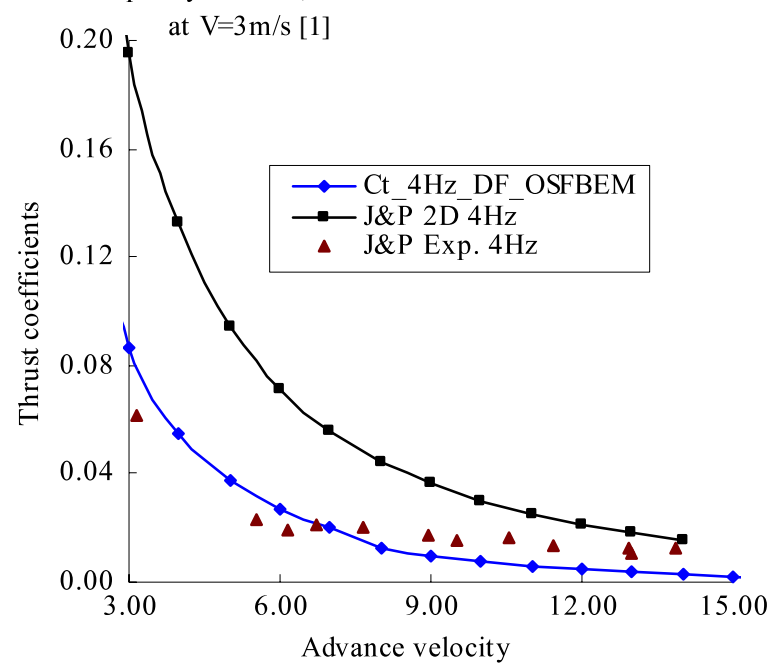

Fig. 4. Thrust coefficients $C_{t}$ of one of the dual foils at a frequency of $4 \mathrm{~Hz}$, estimated maximum AoA: $12^{\circ}$ at $V=3 \mathrm{~m} / \mathrm{s}$, measured [1] versus current predictions.

Thrust coefficients $\mathrm{Ct}$ at a frequency of $6 \mathrm{~Hz}$, estimated maximum AoA: $18^{\circ}$ at $\mathrm{V}=3 \mathrm{~m} / \mathrm{s}$ [1]

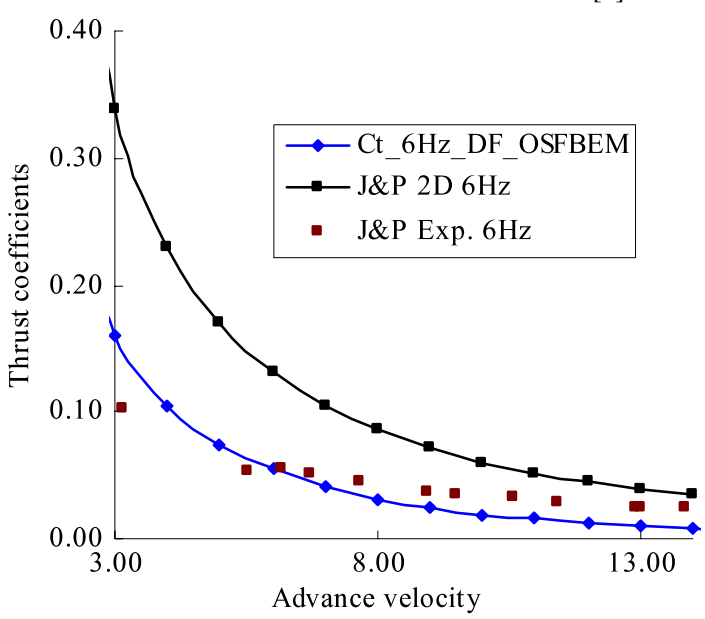

Fig. 5. Thrust coefficients $C_{t}$ at a frequency of $6 \mathrm{~Hz}$, estimated maximum AoA: $18^{\circ}$ at $V=3 \mathrm{~m} / \mathrm{s}$, measured [1] versus current predictions.

would allow a much higher effective AoA before stall occurs. The threshold AoA in this work is taken by experience as $16^{\circ}$.

Fig. 7 shows predicted efficiency of the dual-foil propulsor with three oscillating frequencies.

In Fig. 7, the predicted maximum efficiency is about $65 \%$ and is about the same for all three different frequencies. Liu $[2,3]$ presented an analysis of the effect of various geometry and motion parameters on propulsive efficiency. Parameters used include the closest distance between the dual foils, i.e., wing-in-ground effect on propulsive efficiency.

Figs. 8 and 9 show comparisons between thrust and efficiency, respectively, measured (by Anderson et al. [10]) and predicted by DF_OSFBEM, for a single foil with a standard sinusoidal oscillation using two different sets of oscillation parameters. Brief geometry and motion parameters of the single-foil propulsor are as follows:

- an aspect ratio is assumed to be $A R=7.00$ (this was an $\mathrm{AR}=6.00$ foil equipped with end plates to simulate a $2 \mathrm{D}$ foil in Anderson's work);

- the foil section is NACA0012;

- the foil is in standard sinusoidal motion;
Thrust coefficients $\mathrm{Ct}$ at a frequency of $8 \mathrm{~Hz}$, estimated maximum AoA: $23^{\circ}$ at $\mathrm{V}=3 \mathrm{~m} / \mathrm{s}[1]$

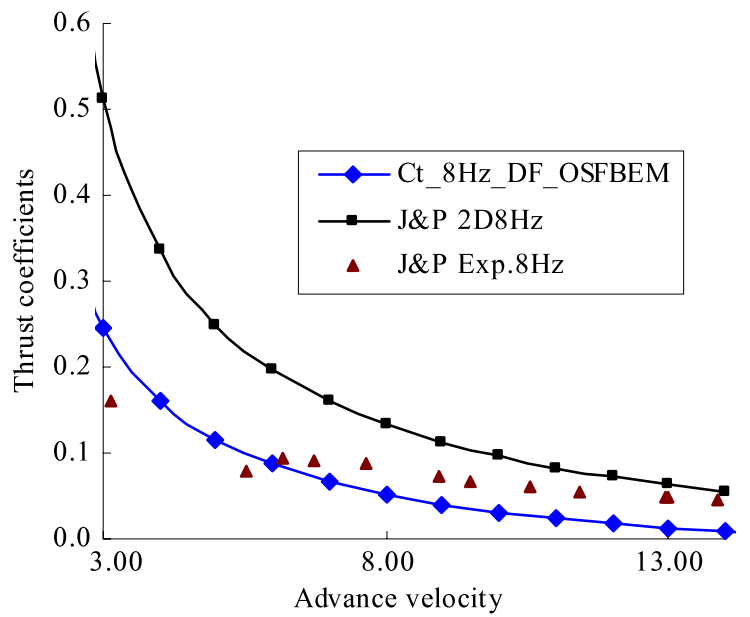

Fig. 6. Thrust coefficients $C_{t}$ at a frequency of $8 \mathrm{~Hz}$, estimated maximum AoA: $23^{\circ}$ at $V=3 \mathrm{~m} / \mathrm{s}$, measured [1] versus current predictions.

Efficiency of one of the dual foils at a frequency of 4 , 6 and $8 \mathrm{~Hz}$

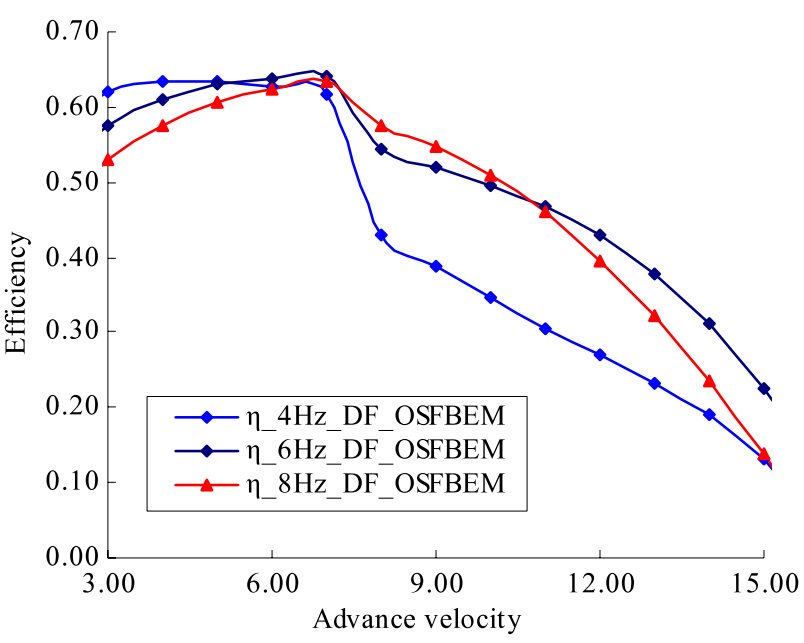

Fig. 7. Efficiency of one of the dual foils at a frequency of 4,6 and $8 \mathrm{~Hz}$.

- the foil's advance speed is set to $3.00 \mathrm{~m} / \mathrm{sec}$ with a pivot axis at $1 / 3$ chord position.

More motion parameters are tabulated in the Table 1.

Figs. 8 and 9 show a general agreement between the measured performance and that predicted by DF_OSFBEM.

We further investigated propulsive characteristics of oscillating foil propulsors in four configurations in terms of efficiency and thrust production. The details of the four configurations are as follows:

- a single-foil propulsor in sinusoidal motion with pitch leading heave $90^{\circ}$ (pitch amplitude $\alpha_{a}=55^{\circ}$, heave amplitude $h_{a}=$ $1.14 C_{r}$ );

- a dual-foil WIG thruster in sinusoidal heave-only counter-phase motion (heave amplitude $h_{a}=0.57 C_{r}$ );

- a single-foil propulsor with simple sinusoidal heave-only motion (heave amplitude $h_{a}=0.57 C_{r}$ );

- a single-foil propulsor with simple sinusoidal heave-only motion (heave amplitude $h_{a}=1.14 C_{r}$ ).

Foils' planform shapes in these four configurations are all rectangular with a span of $8 \mathrm{~m}$ and a chord of $0.7 \mathrm{~m}$. The foil 
Table 1

Geometry and motion parameters for the single, standard sinusoidal-motion oscillating foil.

\begin{tabular}{|c|c|c|c|c|c|c|}
\hline $\begin{array}{l}k=\omega c / 2 / V \text { (definition used } \\
\text { by Anderson et al.) }\end{array}$ & $v(\mathrm{~m} / \mathrm{s})$ & $n$ & $\omega$ & $\operatorname{Re}$ & St & $\begin{array}{l}k=\omega c / V \text { (other common } \\
\text { definition) }\end{array}$ \\
\hline 0.20 & 3.00 & 1.91 & 12.00 & 300000 & 0.12 & 0.40 \\
\hline 0.39 & 3.00 & 3.72 & 23.40 & 300000 & 0.23 & 0.78 \\
\hline 0.50 & 3.00 & 4.77 & 30.00 & 300000 & 0.30 & 1.00 \\
\hline 0.61 & 3.00 & 5.82 & 36.60 & 300000 & 0.36 & 1.22 \\
\hline 0.73 & 3.00 & 6.97 & 43.80 & 300000 & 0.43 & 1.46 \\
\hline 0.83 & 3.00 & 7.93 & 49.80 & 300000 & 0.49 & 1.66 \\
\hline 0.93 & 3.00 & 8.88 & 55.80 & 300000 & 0.55 & 1.86 \\
\hline 1.03 & 3.00 & 9.84 & 61.80 & 300000 & 0.61 & 2.06 \\
\hline 1.30 & 3.00 & 12.41 & 78.00 & 300000 & 0.76 & 2.60 \\
\hline 1.60 & 3.00 & 15.28 & 96.00 & 300000 & 0.94 & 3.20 \\
\hline 1.90 & 3.00 & 18.14 & 114.00 & 300000 & 1.12 & 3.80 \\
\hline
\end{tabular}

Thrust coefficient and efficiency of a rectangular foil with a heave amplitude $=0.75$ chord, pitch amplitdue $=15^{\circ}$ and phase angle $=90^{\circ}$

[10]

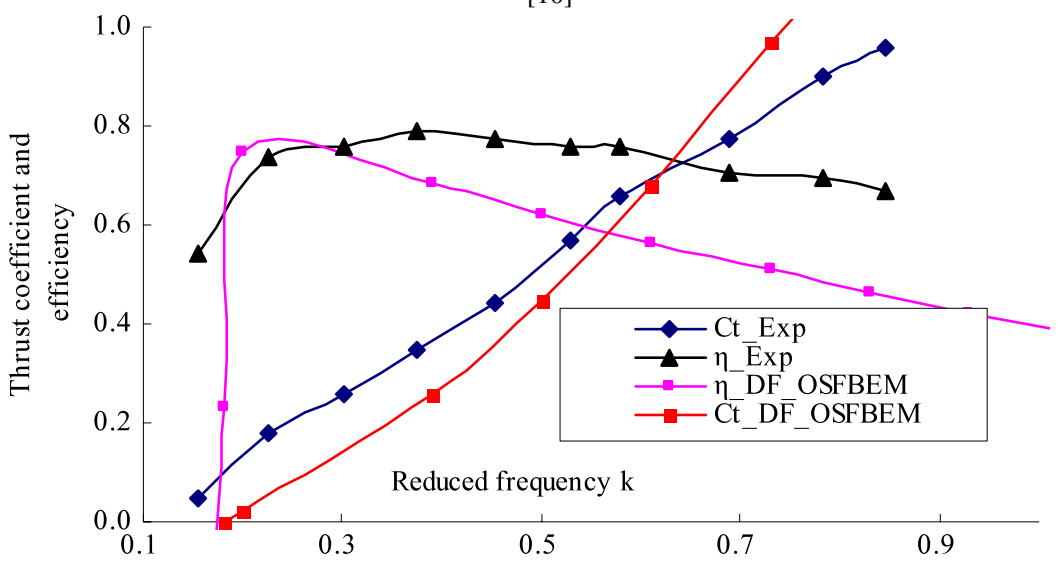

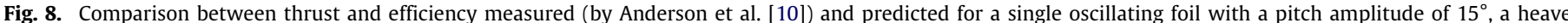
amplitude of $75 \%$ chord and a pitch leading heave phase angle of $90^{\circ}$.

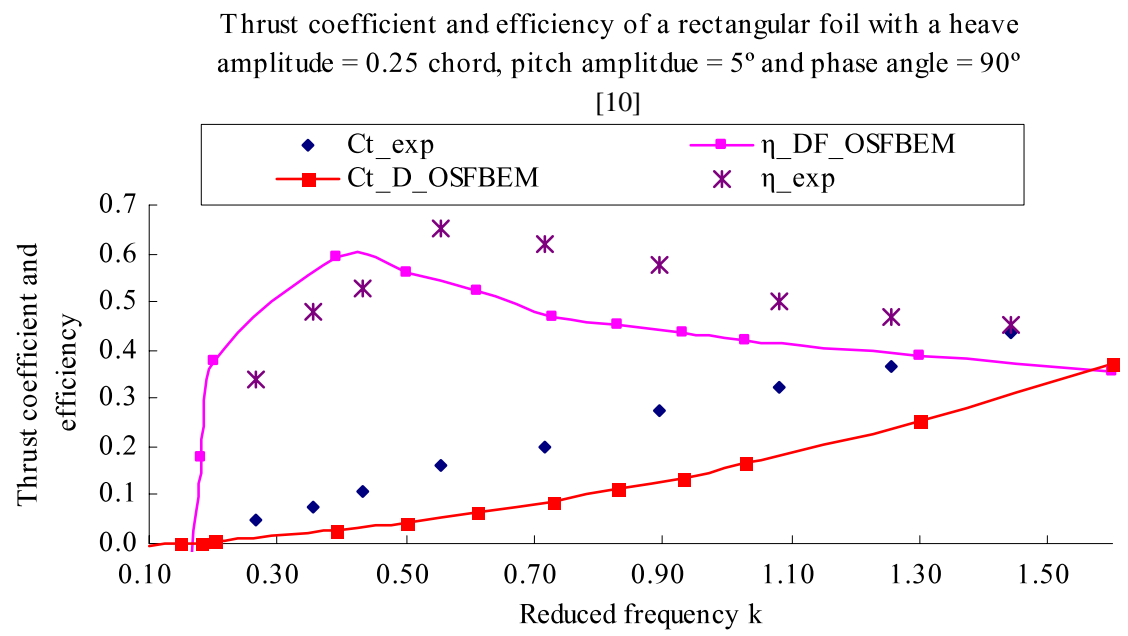

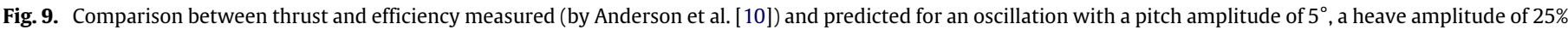
chord and a phase angle between heave and pitch of $90^{\circ}$.

sectional offsets are NACA 63(3)-018 at an oscillating frequency of $1.48 \mathrm{~Hz}$. The path of the pivot axis that is located at $25 \%$ of the chord at the leading edge is governed by the following equations:

$z=h_{a} \cos (\omega t)+z_{0}$

$\alpha=\alpha_{a} \cos (\omega t+\phi)+\alpha_{i n i}$,

where $h_{a}$ is the heave amplitude in meters, $z_{0}$ is the initial vertical location of the pivot axis, $\alpha_{a}$ is the pitch amplitude, $\alpha_{i n i}$ is the initial geometric AoA (zero in this case) and $\phi$ is the phase angle $\left(90^{\circ}\right.$ in this case).

For the dual-foil WIG thrust with half heave amplitude $\left(1.14 C_{r} / 2.00\right)$, heave-only simple sinusoidal motion, the vertical position in time history of the second foil is negative to the first foil in terms of Eq. (1). As the motion is heave only, the pitch amplitude is set to zero.

Figs. 10-12 show comparisons for efficiency and thrust production for four configurations. 


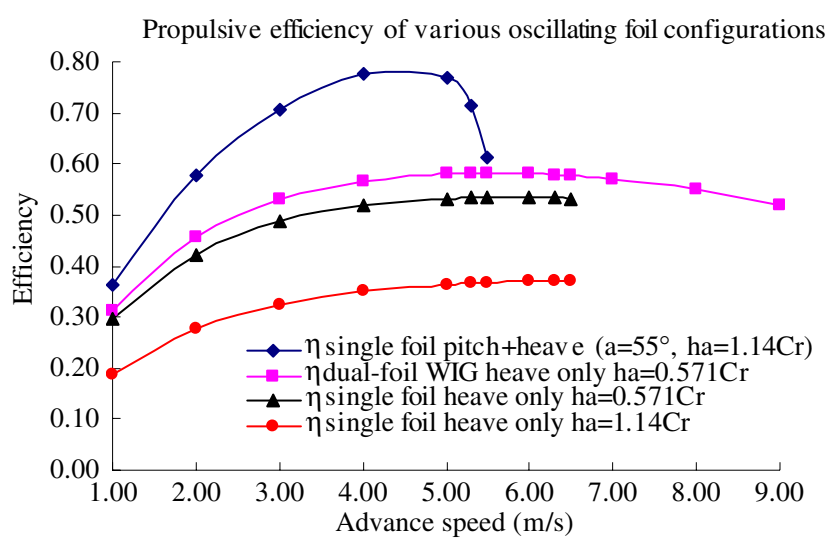

Fig. 10. Comparison for efficiency for four configurations.

Propulsive thrust coefficient of various oscillating foil configurations

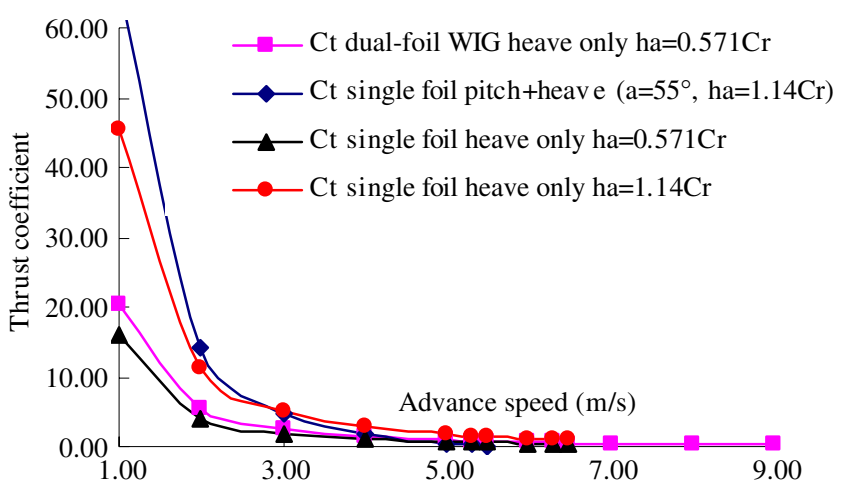

Fig. 11. Comparison for thrust coefficient for four configurations.

Efficiency wise, single-foil propulsors with both pitch and heave produced the highest peak efficiency of about $80 \%$. (Peak efficiency of about $90 \%$ may be possible with some optimization of the motion control.) Dual-foil propulsors with heave-only counter-phase sinusoidal motion produced the second highest peak efficiency (about $60 \%$ ). However, this propulsor maintains a nearly constant efficiency value almost independent of speed. This will be discussed in more detail in the following sections. This velocity independence characteristic could make the propulsor capable of very high speed operation. Single-foil heave-only propulsors produced reduced efficiency throughout the speed range compared with the foils of the dual-foil propulsor. This means that in this configuration, the wing-in-ground effect increased efficiency by around $15 \%$. When the heave amplitude is doubled $\left(h_{a}=1.14 C_{r}\right)$ for the single-foil heave-only propulsor, efficiency decreased about $30 \%$. At a forward speed of $5.00 \mathrm{~m} / \mathrm{s}$, the efficiency of the four propulsors is $77 \%, 58 \%$, $53 \%, 36 \%$, respectively.

At a very low speed of $1.00 \mathrm{~m} / \mathrm{s}$, a single-foil propulsor with both pitch and heave produced the largest thrust. With the increase of speed, thrust coefficient dropped dramatically.

Fig. 12 gives a clearer comparison of thrust in terms of thrust force production.

It can be seen again that the single-foil propulsor with heave and pitch motion produced the highest start-up (sudden acceleration) thrust force. However, its thrust production dropped dramatically with the increase of speed. The thrust production becomes negative at a speed of about $5.50 \mathrm{~m} / \mathrm{s}$. This speeddependent property is the same as the traditional screw propeller. Contrary to combined heave and pitch motion, oscillating foil propulsors with heave-only motion produced almost constant thrust force independent of speed. These heave-only oscillating foil propulsors can operate in a very high speed range, though the efficiency is not the highest.

\section{Similitude establishment between screw propellers and WIG thrusters}

The basic propulsive performance of conventional screw propellers is expressed commonly in terms of nondimensional thrust, torque coefficients and efficiency $(K t-K q-\eta)$ curves versus advance coefficient $J$, the nondimensional speed. Fig. 13 shows a typical set of these curves.

Thrust and torque coefficients are usually described by a set of linear regression polynomials that were extracted from an extensive propeller model test resulting in a propulsion data set [11]. In Fig. 13, torque coefficient curves are omitted as efficiency is a function of torque and thrust coefficient, as $\eta=\frac{J K_{t}}{2 \pi K_{q}}$. In evaluating the propulsion performance, the primary motion and geometry parameters being used are the advance coefficient $J$ and the pitch-diameter ratio, $P_{D}=p / D$, respectively, where $p$ is the pitch at local radius and $D$ is the diameter of the propeller. To further generalize the propulsive performance of screw propellers, we defined a speed-pitch ratio parameter, $G=J / P_{D}$. The performance curve is re-plotted versus $G$ in Fig. 14.

For all five screw propellers of different pitch values, thrust is the maximum and efficiency is zero at zero speed $(G=0.00)$. With the increase of speed, thrust decreases gradually to zero at $G=1.10$ and becomes negative at $G>1.10$. Efficiency increases to its maximum at $G=0.90$ and decreases rapidly to zero at $G=1.10$, becoming negative at $G>1.10$. For any other screw propeller families, the above-mentioned trends normally remain the same, though the critical value of $G$ might shift to left or right. The value of parameter $G$ determines the load condition of a propeller. In the first quadrant operation (i.e. both rotational direction and velocity of advance are positive), a screw propeller operates under the heaviest load condition when $G$ is zero, under the most lightly loaded condition when $G$ value approaches 1.10 , and under a negative load condition when Gvalues are greater than 1.10 (turbine power generator mode).

Here we are going to examine two primary propulsive characteristics of the WIG thrusters: (1) the speed-geometry similitude and scalability, comparable to traditional screw propellers and (2) the equivalent screw propeller pitch ratio corresponding to the heave amplitude of WIG thrusters. With respect to geometry, we defined the span $S_{p}$ as the reference length corresponding to propeller diameter $D$. To examine the geometry-speed scalability, we further defined a thrust production factor, $f_{t p}=n \times D \approx n \times S_{p}$, where $n$ is the rotation speed in revolution per second and $D$ is the diameter of the propeller in meters, based on the formulation of advance coefficient $J$ (see Table 1 ). With the same geometry shape, propellers having the same values of $f_{t p}$ should produce about the same thrust coefficient when the Reynolds number effect is insignificant (for marine transportation vehicles the Reynolds number is usually greater than $10^{6}$ in which case the Reynolds number effect could be negligible). This means that the thrust coefficient produced by a propeller with a diameter of $D=1 \mathrm{~m}$ at $n=10 \mathrm{rps}$ should be about the same as that from a propeller of the same geometry with a diameter of $D=10 \mathrm{~m}$ at $n=1 \mathrm{rps}$. In the meantime, for propellers with the same geometry shape, the larger the values of $f_{t p}$, the higher the thrust coefficient. It is noted that a propeller having a high thrust coefficient does not necessarily imply a high thrust force production as thrust force is proportional to $n^{2}$ and $D^{4}$, respectively.

To begin with the examination of the two key propulsive characteristics, a similitude analysis was also made. Based on propeller theory and data in Fig. 14, we established the 
Propulsive thrust force of various oscillating foil configurations

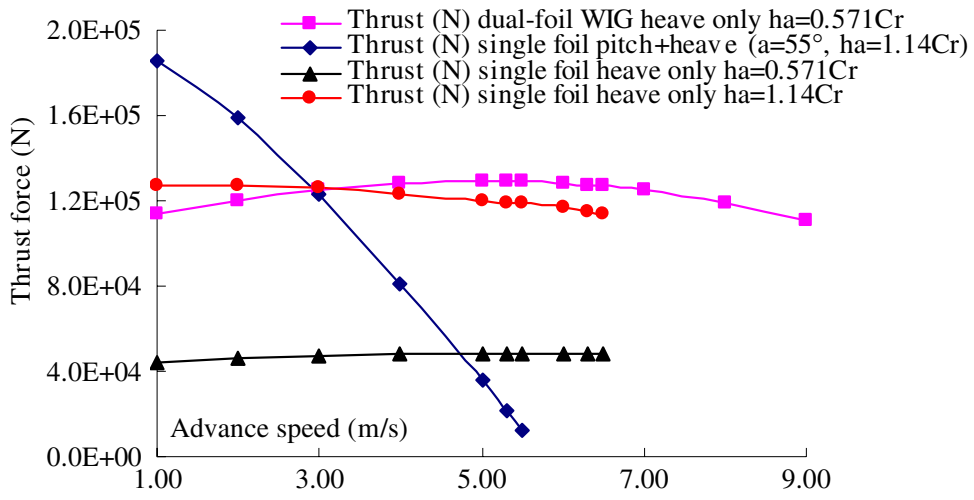

Fig. 12. Comparison of thrust forces for four configurations.

Table 2

Similitude parameters of WIG effect thruster corresponding to screw propeller.

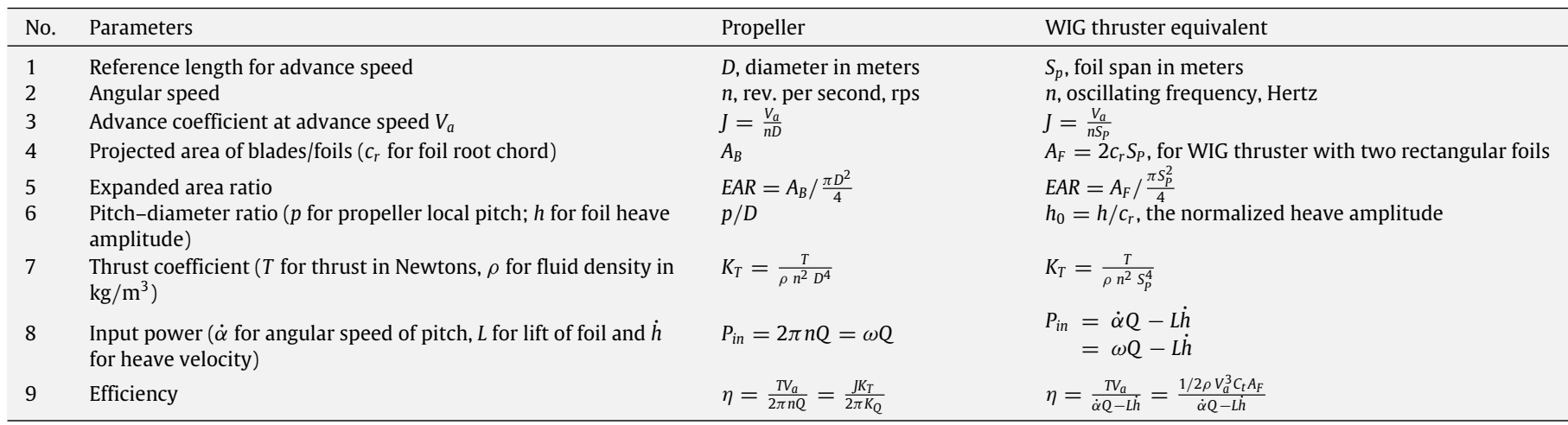

corresponding propulsive geometry and motion parameters for WIG effect thrusters and these are listed in Table 2.

For WIG thrusters, the span of the wing is chosen as the reference length. For marine propulsor design, the length of the span of the foil is similar to the diameter of the propeller when considering the space for propulsor installation at the stern of a ship. That is why the chord length was not used as reference length. For propellers, the expanded area ratio of a propeller is a function of number of blades, local chord length and the hub diameter. For propellers of the same shape, the expanded area ratio is constant for any propeller size. Therefore, there is no need for the span of foil to reflect propeller hub radius.

To determine the speed-geometry similitude and scalability of the WIG thrusters versus screw propellers, we obtained the efficiency and thrust coefficient of WIG thrusters of four different geometry dimensions with the same thrust production factor $f_{g s}=$ 6 , by using the time-domain unsteady panel method, DF_OSFBEM, as a virtual wind/cavitation tunnel. The geometry and motion parameters for these WIG thrusters of four different sizes are given in Table 3.

Fig. 15 shows the similitude and scalability of the thrust and efficiency of the WIG thrusters of four different sizes with the same equilibrium position factor $z_{0}=Z / C_{r}=0.70$.

It indicates that nearly identical thrust coefficient and efficiency are produced by the WIG thrusters of four different sizes at the same heave amplitude factor in a range of $J$ from 0.00 to 5.00, because their thrust production factor $f_{\text {tp }}$ is the same. There is only a slight drop in the thrust coefficient when the advance coefficient $J$ reaches 5.00. For WIG thrusters, thrust and efficiency drop very slowly and gradually, deteriorating when the speed is substantially high $(J>2)$. In fact, with the same shape but different sizes, production of the thrust coefficient and efficiency should be about

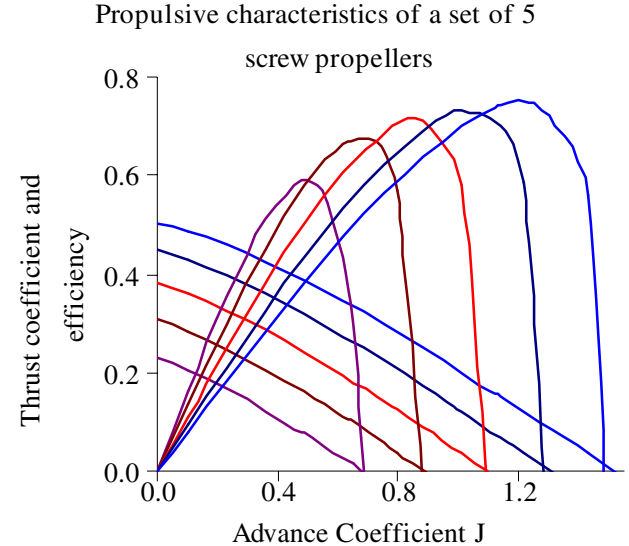

Fig. 13. Thrust coefficient and efficiency versus advance coefficient $J$ of propellers with five different pitch-diameter ratios.

the same, as long as the thrust production factor $f_{t p}$ is the same when the Reynolds number effect is negligible and the flow has no severe cavitation in water. The discrepancy in the thrust coefficient at large $J$ values between the different sizes is due to the Reynolds number effect (the numerical code estimated the skin friction as a function of Reynolds number). For marine applications, the critical Reynolds number of a WIG thruster is usually $1.0 \times 10^{6}$ or greater. This critical value in fact falls into the range of practical marine applications. However, for aerial applications, especially for micro aerial vehicles, both the light fluid density and the small foil size can result in a much smaller $R e$ value; thus stall and separation could be significant at low speeds of advance. The foil section used in the current work has a symmetric NACA0014 section; thus, the 
Table 3

Geometry and motion parameters of the four WIG thrusters.

\begin{tabular}{|c|c|c|c|c|c|c|c|c|}
\hline $\begin{array}{l}\text { Oscillating frequency } \\
n(\mathrm{~Hz})\end{array}$ & $\begin{array}{l}\text { Chord } \\
C_{r}(\mathrm{~m})\end{array}$ & $\begin{array}{l}\text { Span } \\
S_{P}(\mathrm{~m})\end{array}$ & $\begin{array}{l}\text { Thrust production factor } \\
S_{p} * n f_{g s}\end{array}$ & $\begin{array}{l}\text { Total area } \\
A_{F}\left(\mathrm{~m}^{2}\right)\end{array}$ & $\begin{array}{l}\text { Aspect } \\
\text { ratio } A R\end{array}$ & $\begin{array}{l}\text { Area } \\
\text { ratio } E A R\end{array}$ & $\begin{array}{l}\text { Heave } \\
\text { factor } h\end{array}$ & $\begin{array}{l}\text { Position factor } \\
Z\end{array}$ \\
\hline 10.00 & 0.10 & 0.60 & 6.00 & 0.12 & 6.00 & 0.424 & $0.60 C_{r}$ & $0.70 C_{r}$ \\
\hline 2.00 & 0.50 & 3.00 & 6.00 & 0.30 & 6.00 & 0.424 & $0.60 C_{r}$ & $0.70 C_{r}$ \\
\hline 1.00 & 1.00 & 6.00 & 6.00 & 12.00 & 6.00 & 0.424 & $0.60 C_{r}$ & $0.70 C_{r}$ \\
\hline 0.50 & 2.00 & 12.00 & 6.00 & 48.00 & 6.00 & 0.424 & $0.60 C_{r}$ & $0.70 C_{r}$ \\
\hline
\end{tabular}

Table 4

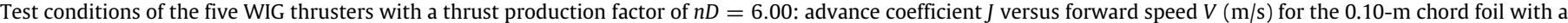
heave amplitude of $1.50 C_{r}(0.15-\mathrm{m})$.

\begin{tabular}{|c|c|c|c|c|c|c|c|c|c|c|}
\hline Speed V (m/s) & 0.10 & 0.20 & 0.40 & 0.80 & 1.60 & 3.20 & 6.40 & 12.80 & 25.60 & 51.20 \\
\hline Speed $V(\mathrm{~km} / \mathrm{h})$ & 0.36 & 0.72 & 1.44 & 2.88 & 5.76 & 11.52 & 23.04 & 46.08 & 92.16 & 184.32 \\
\hline Advance coefficient $J$ & 0.017 & 0.033 & 0.067 & 0.13 & 0.267 & 0.53 & 1.07 & 2.13 & 4.27 & 8.53 \\
\hline Reduced frequency $K$ & 62.83 & 31.41 & 15.70 & 7.85 & 3.92 & 1.96 & 0.98 & 0.49 & 0.245 & 0.123 \\
\hline $\operatorname{Re}$ for water $\left(/ 10^{3}\right)$ & 628.40 & 628.64 & 629.59 & 633.39 & 648.37 & 705.11 & 896.88 & 1425.90 & 2635.98 & 5158.41 \\
\hline $\operatorname{Re}$ for air $\left(/ 10^{3}\right)$ & 94.89 & 94.92 & 95.07 & 95.64 & 97.90 & 106.47 & 135.43 & 215.31 & 398.03 & 778.92 \\
\hline
\end{tabular}

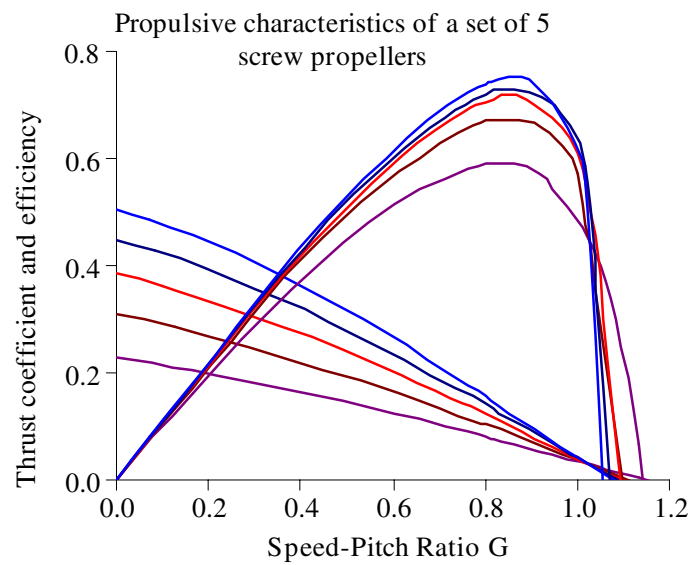

Fig. 14. Thrust coefficient and efficiency versus speed-pitch ratio of propellers with five different pitch-diameter ratios.

Scalability of thrust coefficient and efficiency from 5 WIG thrusters with $\mathrm{AR}=6, \mathrm{z}=0.7 \mathrm{Cr}$ and $\mathrm{h}=0.6 \mathrm{Cr}$

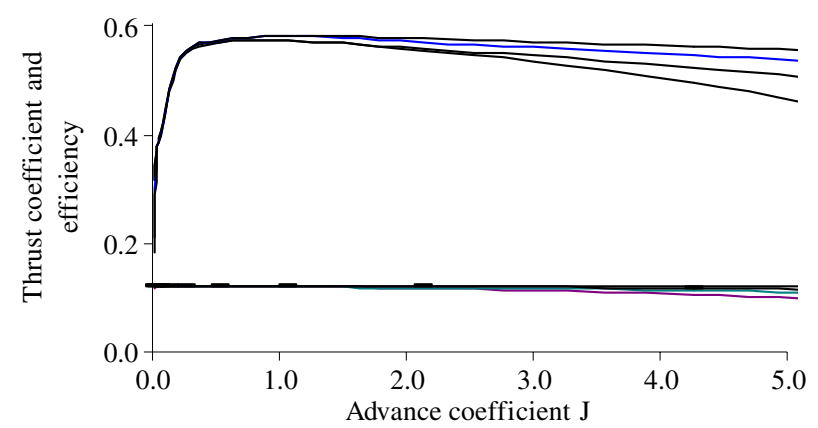

Fig. 15. Geometry scalability of thrust coefficient versus $J$ for five WIG thrusters with a heave amplitude $h=0.60 C_{r}$ and an equilibrium position $z=0.70 C_{r}$.

minimum gap is then $2\left(10 \%-\frac{14 \%}{2}\right) C_{r}=6 \% C_{r}$. The principal motion parameters, i.e., the advance coefficient $J$ versus speed, along with the $R e$ for water and air for a $0.10-\mathrm{m}$ chord foil of an aspect ratio 6 , are listed in Table 4 as an example.

In Table 4, Re, the Reynolds number was defined using the root chord $C_{r}$ as the reference length, via $R e=\frac{\sqrt{\left(2 \pi n h_{0} C_{r}\right)^{2}+V^{2}} C_{r}}{v}$, where $v$ is kinematic viscosity. At $20^{\circ} \mathrm{C}, \operatorname{Re}$ is about $1.0^{\nu} \times 10^{6}$ in water and $1.51 \times 10^{5}$ in air, respectively. The Re in air is about $1 / 6$ of that in water at sudden acceleration or bollard-pull (zero speed) condition; this indicates earlier stall and separation, and hence the possibilities of a dramatic drop in thrust. This becomes critically important if the size of the WIG thrusters is small, especially for micro aerial vehicles. A self-adjusted pitch [3] with a passive damping by enabling the shaft with torsional flexibility will reduce the effective AoA substantially at very low speed and this will result in a substantial increase in thrust and efficiency.

To examine the propulsive performance scalability in terms of speed and pitch variables, we used five different heave amplitudes for the WIG thrusters with a constant $f_{t p}$ value of 1.8 . This value of $f_{t p}$ simulates the marine propellers above at a realistic speed range. These heave amplitudes correspond to the pitch-diameter ratio of these screw propellers of $P_{D}=0.60,0.80,1.00,1.20$ and 1.40 , respectively. For each heave amplitude value, the minimum distance between the nose-tail lines of the two foils was limited to $20 \%$ of the foil's root chord. That is, the heave amplitude was taken as $10 \%$ of the chord less than the height of $z$, which is between the equilibrium position and the plane of geometry (Fig. 1).

The pitch of conventional propellers is the geometric distance it moves per revolution. The difference between the geometry distance and the travel distance, along with the influence of induced axial and tangential velocities, determines the effective AoA. The effective AoA is directly proportional to the lift and hence thrust production of a foil (or blade) section. For WIG thrusters with heave-only motion, the effective AoA is proportional to their heave amplitude and this heave amplitude therefore could be treated as the equivalent geometry parameter to the pitch that contributes to thrust loading of screw propellers.

To examine the similarity of thrust and efficiency versus advance coefficient and pitch ratio, we took the span of the foils to be equal to the diameter of the propellers, at $S_{p}=0.60 \mathrm{~m}$. The expanded area ratio of the propeller is determined by the total area of two foils divided by the area of the propeller disk of $D=S_{p}$ (see Table 1). For a WIG thruster with two rectangular foils of $0.10 \mathrm{~m}$ by $0.60 \mathrm{~m}$ resulting in an aspect ratio of 6 , the equivalent expanded area ratio to the $0.60 \mathrm{~m}$-diameter crew propeller is 0.4244 . To obtain the heave amplitudes corresponding to the pitch values of screw propellers, we first determined the value of the required thrust from WIG thrusters. The required thrust value was taken from the propeller's thrust at the highest efficiency, which is usually chosen as the vehicle's service speed. The highest efficiency occurs at the thrust loading point factor at about $G=0.90$ for the family of propellers being considered. The thrust at this point is about $1 / 3$ of the maximum thrust of these propellers (at $G=$ 0.0.). A number of runs were performed to obtain the relationship between heave amplitude and thrust of WIG thrusters and the relationship between pitch and thrust of propellers. Using a foil size of a $0.10 \mathrm{~m}$ chord and a span of $0.60 \mathrm{~m}$, we calculated the thrust and efficiency for heave amplitude factors of $h_{0}=0.40-0.80$. The relationships between the heave amplitude of the WIG thruster 


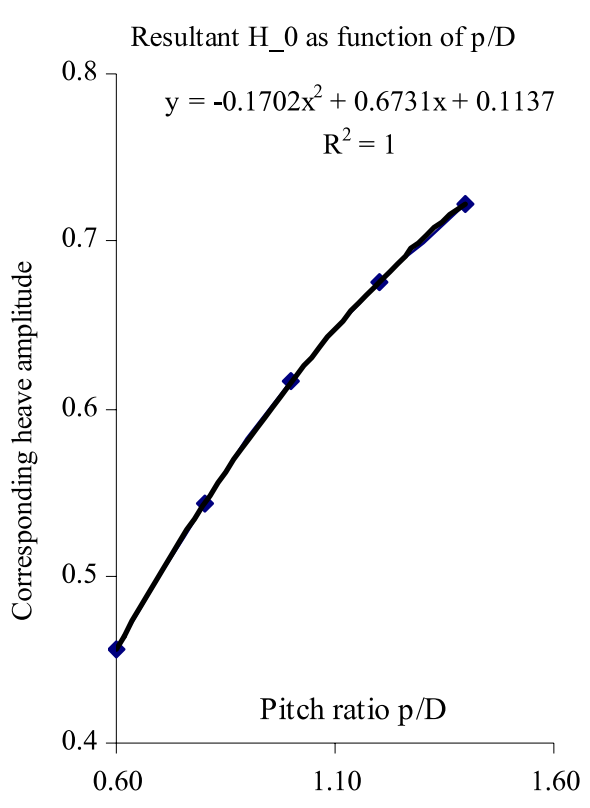

Fig. 16. Heave amplitude as a function of corresponding pitch-diameter ratio.

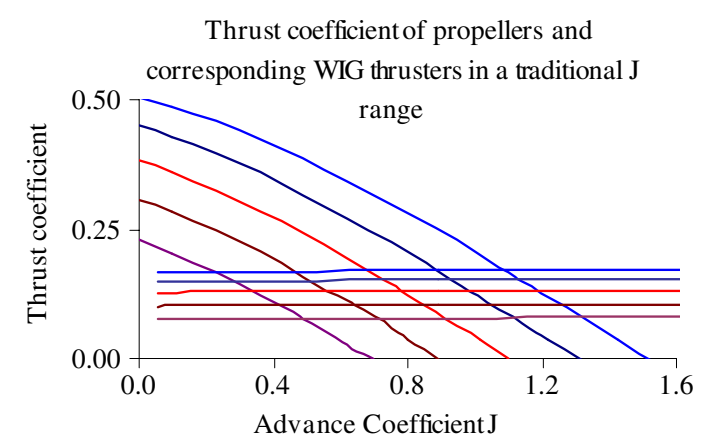

Fig. 17. Thrust production from five WIG thrusters and propellers of corresponding pitch values, in a conventional range of advance coefficient $J$.

and the pitch-diameter ratio of propellers were obtained and are shown in Fig. 16.

With the trend line equation, for this particular WIG thruster geometry, heave amplitude values corresponding to pitchdiameter ratio were obtained as $h_{0}=0.4562,0.5433,0.6169$, 0.6759 and 0.7226 to correspond to $P_{D}=0.60,0.80,1.00,1.20$ and 1.40. The equilibrium positions of the foils for the heave amplitude value of the five WIG thrusters are the same, taken as $z_{0}=h_{0}+0.1$.

Figs. 17 and 18 show the propulsive characteristics of the conventional propellers and the WIG thrusters of corresponding pitch-diameter ratios, in a conventional and extended $J$ range, respectively.

Thrust produced from conventional propellers shows a strong speed dependency and drops dramatically with the increase of the speed. Operating in a rotational motion in the first quadrant (positive rotation and advance speed), conventional screw propellers produce a thrust that is proportional to the effective AoA and the maximum thrust production occurs only at the lowest speed at $J=0.0$. With the increase of the speed of advance, the value of the effective AoA and hence the thrust drops dramatically to zero and then goes negative. For such a propeller with a diameter $D=0.60 \mathrm{~m}$ or a WIG thruster with a span of $S_{p}=0.60 \mathrm{~m}$, when the rotational speed or oscillating frequency is taken as $n=3 \mathrm{rps}$ and hence the thrust production factor is $f_{t p}=1.8$, the corresponding range of speed of advance for $J=0.00-1.55$ is $0.00-2.80 \mathrm{~m} / \mathrm{s}$, i.e., $0.00-10.00 \mathrm{~km} / \mathrm{h}$. This means that the maximum possible speed that the $0.6 \mathrm{~m}$ propellers could
Thrust coefficient of propellers and WIG thrusters

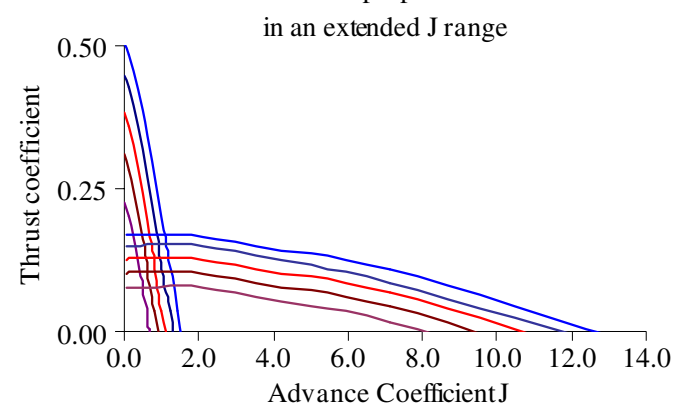

Fig. 18. Thrust production from five WIG thrusters and propellers of corresponding pitch values, in an extended range of advance coefficient $J$.

Efficiency of propellers of 5 pitch ratios and corresponding WIG thrusters in a traditional $\mathrm{J}$

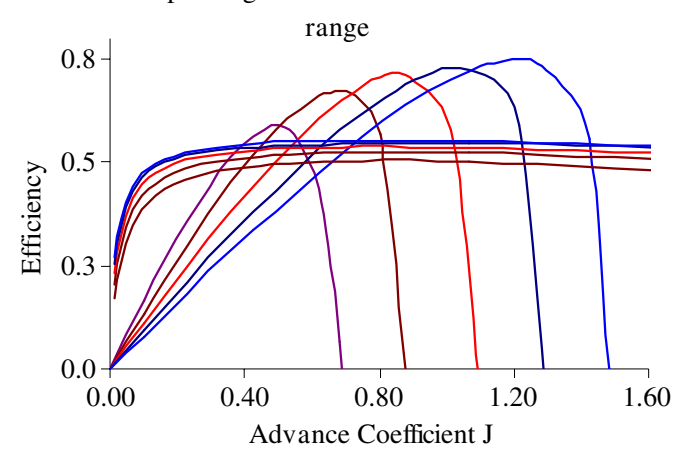

Fig. 19. Efficiency versus advance coefficient $J$ in the traditional speed range.

reach is $10 \mathrm{~km} / \mathrm{h}$. However, the maximum attainable speed for WIG thrusters is $J=12.0$, i.e., $78 \mathrm{~km} / \mathrm{h}$. Unlike propellers, the thrust production from a WIG thruster is nearly independent of the speed in the whole speed range for propellers (except a small speed portion where the speed is close to zero). Operating in heave motion only, WIG thrusters will always produce a positive thrust in an ideal fluid for any speed. In the propeller speed range, the thrust production of a WIG thruster is effectively constant. As mentioned, the propulsive performance comparison is based on equal thrust production at a speed where the highest efficiency occurs. With this assumption, either for a propeller or the equivalent WIG thruster of the same equivalent pitch, a vehicle can obtain the same design speed with either propulsor, though a vehicle propelled by a WIG thrust will accelerate relatively slowly. However, with the speed independent characteristics, a WIG thruster will continue to provide positive thrust at speeds of three to six times the maximum attainable speeds of a screw propeller.

Figs. 19 and 20 show a similar trend for efficiency. Though the equivalent WIG thrusters achieve lower peak efficiency, it again shows a speed independency, as it does for thrust production, in the conventional speed range.

With the increase of the speed, the skin friction drag becomes more significant, having a large deduction on the overall thrust and hence the efficiency. In an ideal fluid, the efficiency and thrust of WIG thrusters are constant, but thrust and efficiency will decrease dramatically for propellers, regardless of whether the fluid is ideal or not, because the AoA becomes diminished or negative with the increase of the speed of advance. It is noted also that when the WIG thruster is designed to produce the same amount of thrust at a specific design speed, its maximum attainable efficiency is $10-15 \%$ lower than the equivalent screw propellers.

To examine thrust power characteristics, the maximum achievable thrust power for the five propellers and corresponding WIG thrusters is plotted in Figs. 21 and 22. 


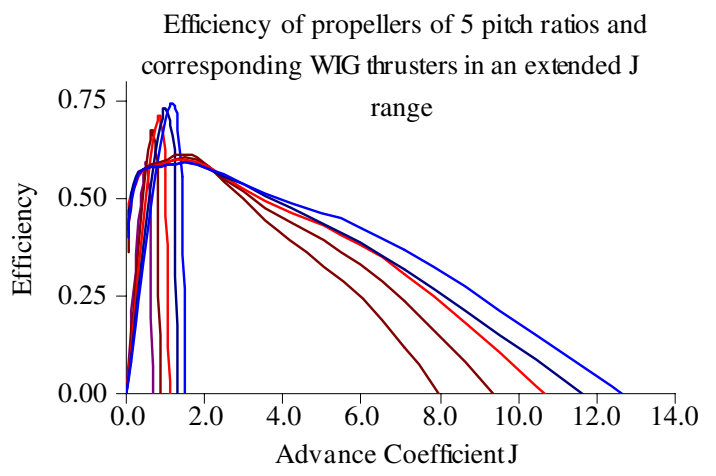

Fig. 20. Efficiency versus advance coefficient $J$ in an extended speed range.

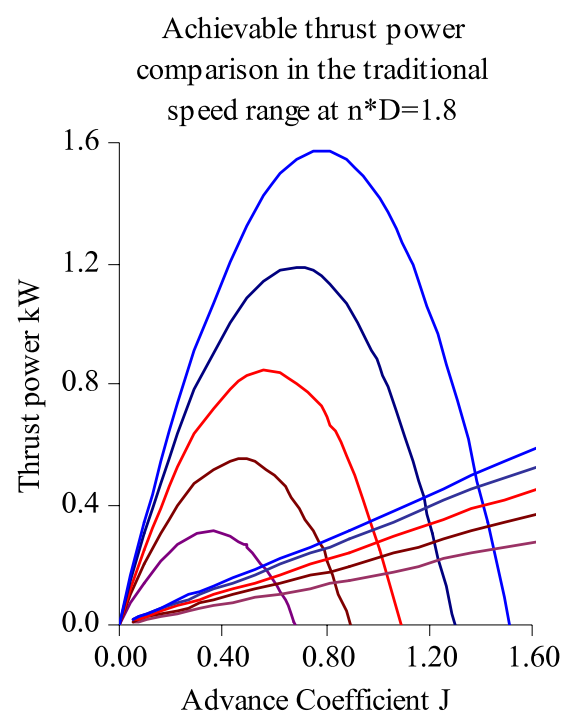

Fig. 21. Maximum attainable thrust power of the traditional propellers and the WIG thrusters in the traditional speed range.

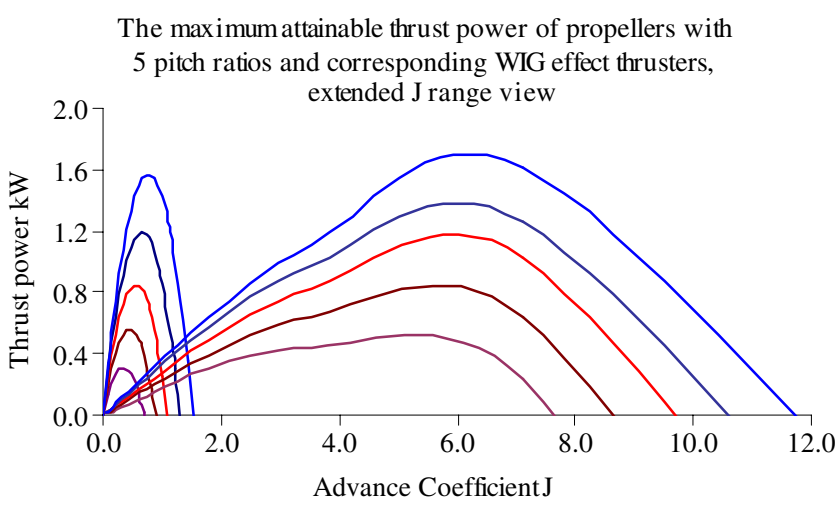

Fig. 22. Maximum attainable thrust power of the traditional propellers and the WIG thrusters in the extended speed range.

Similar to thrust and efficiency, thrust power production of screw propellers also has a very narrow applicable speed range with a limit of $J<2.00$. For propellers, the maximum thrust power usually occurs at about $J=\frac{1}{2} P_{D}$. The maximum achievable thrust power for propellers climbed and dropped steeply in the narrow speed range, while that of the WIG thrusters reached its peak value at a much larger advance coefficient $J$ at about 6.00 (corresponding to $38.00 \mathrm{~km} / \mathrm{h}$ for the $S_{p}=D=0.60 \mathrm{~m}$ case).

For a WIG thruster with the same span as the diameter of the equivalent propeller, the maximum attainable thrust power is about the same as a propeller. As is known, one of the most
Effect of cavitation on thrust coefficient of WIG

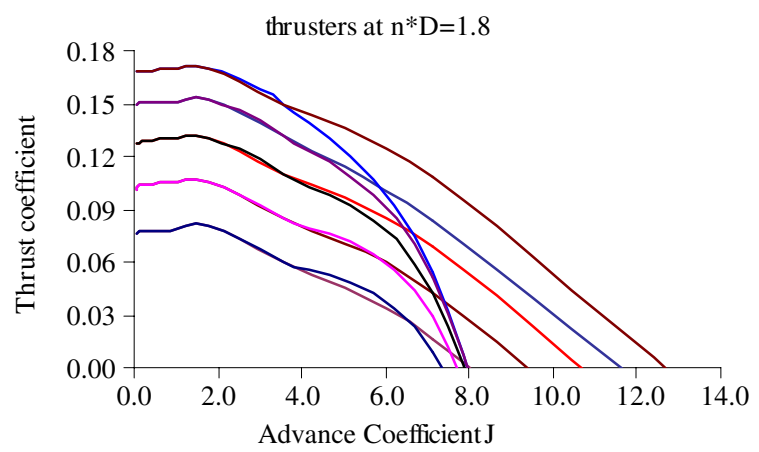

Fig. 23. Effect of cavitation on thrust coefficient and efficiency from the WIG thrusters in the extended speed range.

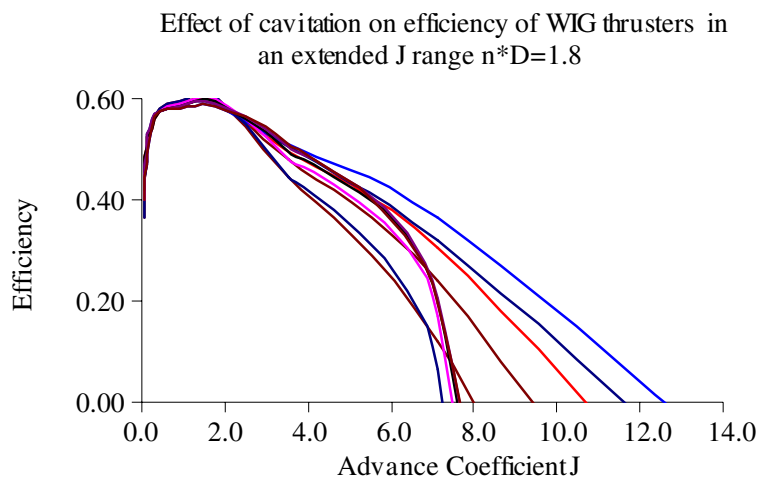

Fig. 24. Effect of cavitation on efficiency from the WIG thrusters in the extended speed range.

challenging problems in conventional propeller design is that the propeller has only one optimal operation speed in terms of efficiency and thrust production. In propeller design, requirements for both speed and thrust production have to be met. For WIG thrusters, the requirement is mainly for thrust production.

It should be noted that in WIG propulsor design the required maximum deliverable power would be substantially larger than that for a propeller, because the thrust production of a WIG thruster is sinusoidal or in the form of pulse. The maximum required deliverable power for a WIG thruster in this study should be about $\sqrt{2}$ times of the mean power.

The above discussions have not addressed cavitation. In water, especially at high speed, cavitation might be so severe as to degrade substantially the performance of WIG thrusters. The effect of cavitation on thrust, efficiency and the maximum attainable thrust power are shown in Figs. 23-25. For thrusters with a thrust production factor of 1.80 , all three properties started to drop at $J>4.00$ and diminished at about $J=8.00$. It suggests that the best speed range for a WIG thruster for marine application is between $2.00<J<6.00$.

A similar investigation of powering characteristics of the WIG thruster revealed that this has not been studied before. With its unique speed-independent property in terms of both thrust and efficiency, the WIG thruster could be promising for much higher speed applications. In the design of conventional propellers, fixed pitch propellers have only one optimum speed, but WIG thrusters can be pre-manufactured in various sizes for different thrust production requirements. In other words, when designing propellers, both the thrust production and efficiency at the design speed are two primary variables to consider; for WIG thrusters, only thrust production is the primary design factor. With the technology advances in linear motors with low mechanical loss, 


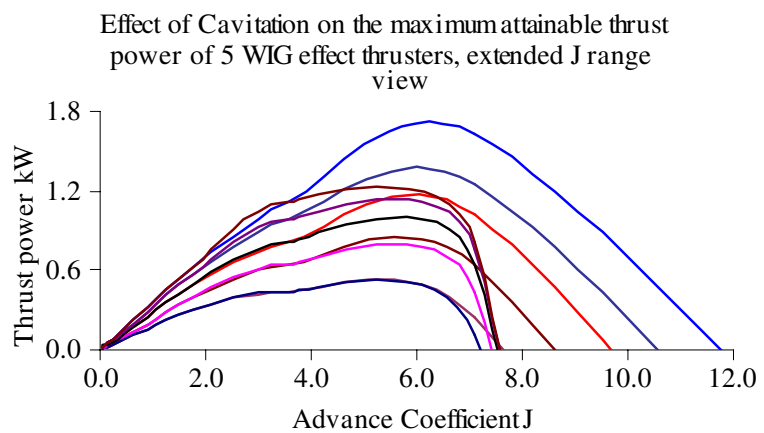

Fig. 25. Effect of cavitation on the maximum attainable thrust power from the WIG thrusters in the extended speed range.

a higher overall efficiency of WIG thrusters is attainable due to the absence of the transformation from rotation to translation. Though further experimental tests to validate WIG thrusters are necessary for the development of the new propulsion device, WIG thrusters appear to be an alternative to the screw propeller in some application cases, especially for high speed vehicles and for high manoeuvrability requirements.

\section{Conclusions}

A comparative study between a family of traditional screw propellers and a family of dual-foil, counter-phase WIG thrusters was carried out. In the study, a similitude system for motion and geometry parameters was established for WIG thrusters corresponding to those of propellers. A thrust loading factor $G$ and thrust production factor $f_{t p}$ were established and used to examine the scalability and geometric similarity of propulsion characteristics of both propellers and WIG thrusters. As the effective AoA, and hence the thrust contribution of a propeller blade section, is mainly determined by a combination of the pitch and the advance speed, propulsion of screw propellers has a very strong speed dependency. This speed dependency indicates that they produce the maximum thrust at $G=0.00$, zero thrust at about $G=1.10$ and negative thrust at about $G>1.10$. This propulsion behavior requires the design of the propeller to meet both required thrust and efficiency at only one fixed speed point. To further compare the propulsive performance between the WIG thrusters and screw propellers, the equivalent heave amplitudes were obtained for the family of WIG thrusters to correspond to the family of screw propellers. It was found that an outstanding propulsion characteristic of WIG thrusters is their independence of speed of advance. This suggests that only thrust production of a WIG thruster needs to be considered in design. Another distinguishing propulsion characteristic of WIG thrusters is their much higher speed capability, about three to six times the speed of advance capability of screw propellers. While the speed independence of WIG thrusters is unlimited in an ideal fluid, its high speed applicability is constrained by cavitation in water and compressibility in air, and by viscous effect in both air and water.

\section{Acknowledgements}

We thank National Research Council of Canada for its continuing support to the project. We are also grateful to the Institute of Mechanics, the Chinese Academy of Sciences (CAS), the Hong Kong K.C. Wong Education Foundation, and the Chinese National Science Foundation for providing the first author with the opportunities for collaboration visits. Thanks to IOT-NRC colleague, Mr. Derek Yetman who performed a proofread of the manuscript.

\section{References}

[1] Jones KD, Plazter MF. An experimental and numerical investigation of flapping-wing propulsion. AIAA J Paper 1999;99-0995:13 p.

[2] Liu P. Propulsive performance of a twin-rectangular-foil propulsor in a counter-phase oscillation. J Ship Res 2005;49(3):207-14

[3] Liu P. Oscillating foil propulsion system. US Patent no. 6,877,692. 2005.

[4] Wu TY. Hydromechanics of swimming propulsion. Part 1. Swimming of a twodimensional flexible plate at variable forward speeds in an inviscid fluid. J Fluid Mech 1971;46(part 2):337-55.

[5] Liu P. A time-domain panel method for oscillating propulsors with both spanwise and chordwise flexibility. Ph.D. thesis, Memorial University of Newfoundland; 1996. 226p.

[6] Liu P, Bose N. Propulsive performance from oscillating propulsors with spanwise flexibility. Proc R Soc Lond 1997;453:1763-1770A.

[7] Wang T, Liu P, Yang G. Preconditioning numerical method and performance investigation of plunging dual foils (1). In: Proceedings of NVTA'2007, the 9th national vibration technology and application. 2007.

[8] Wang T, Liu P, Yang G. Preconditioning numerical method and performance investigation of plunging dual foils (2). In: Proceedings of NVTA'2007, the 9th national vibration technology and application. 2007.

[9] Katz J, Plotkin A. Low-speed aerodynamics: from wing theory to panel methods. McGraw-Hill; 1991.

[10] Anderson JM, Streitlien1 K, Barrett1andm K, Triantafyllou1 K. Oscillating foils of high propulsive efficiency. J Fluid Mech 1998;360:41-72.

[11] Observed MWC, Oossanen Pvan. Further computer-analyzed data of the Wageningen B-screw series. Int Shipbuild Progr 1975;ISP-22:251-62. 